05.1

\title{
Анализ водородоустойчивости алюминиевых сплавов
}

\author{
(ㄱ Д.А. Индейцев, Е.В. Осипова \\ Институт проблем машиноведения РАН, Санкт-Петербург, Россия \\ ฯ E-mail: elena.vl.osipova@gmail.com
}

Поступило в Редакцию 28 сентября 2020 г.

В окончательной редакции 28 сентября 2020 г.

Принято к публикации 7 ноября 2020г.

Методами квантовой химии установлено, что вольфрам и рений являются наиболее эффективными добавками, повышающими водородоустойчивость алюминия. Показано, что W и Re сильно сжимают алюминий, но в то же время имеют достаточно большой ковалентный радиус. Кроме того, каждый атом W и Re образует устойчивые химические связи с 12 атомами Al. B результате атомы W и Re сильно связывают атомы $\mathrm{Al}$, существенно увеличивая энергию образования вакансий и замедляя процесс водородного охрупчивания. С помощью метода функционала плотности рассчитаны все основные физикомеханические свойства наиболее водородоустойчивого соединения алюминия $\mathrm{WReAl}_{24}$.

Ключевые слова: водородное охрупчивание, метод функционала плотности, алюминиевые сплавы.

DOI: 10.21883/PJTF.2021.04.50638.18562

Алюминий и алюминиевые сплавы являются основным материалом при разработке конструкционных элементов в современном машиностроении, особенно в аэрокосмической и автомобильной промышленности [1]. Алюминий и интерметаллиды на основе алюминия являются очень перспективными материалами для водородной энергетики как при расщеплении воды [2], так и при использовании гидридов для аккумулирования водорода [3]. Однако использование алюминия в этих областях все еще ограничено из-за процесса водородного охрупчивания алюминия и его сплавов, особенно под действием механической нагрузки [4]. Поэтому поиск легирующих добавок к алюминию, замедляющих процесс водородного охрупчивания, и создание тем самым водородоустойчивых алюминиевых сплавов являются актуальной задачей [5]. В работе [6] был установлен механизм охрупчивания чистого алюминия водородом. Атом алюминия, который окружен несколькими атомами водорода в октаэдрических пустотах, гораздо легче перемещается в междоузлие (тетраэдрическую пустоту) по сравнению с чистым алюминием. Поэтому в алюминии, в котором растворен водород, вакансии образуются гораздо быстрее, что, согласно флуктуационной теории разрушения Журкова [7], приводит к резкому сокращению времени растрескивания, особенно под действием механической нагрузки [6]. Настоящая работа посвящена нахождению добавок к алюминию, существенно замедляющих процесс водородного охрупчивания, методами квантовой химии с использованием обобщенного градиентного функционала плотности РВE [8].

Основываясь на флуктуационной теории охрупчивания алюминия [6], можно назвать три необходимых свойства, которыми должны обладать искомые добавки к алюминию, чтобы быть оптимальными с точки зрения водородоустойчивости. Во-первых, добавки должны сжимать алюминий, уменышая расстояние между атома- ми алюминия. В чистом алюминии расстояние между ближайшими атомами равно $2.86 \AA$, что слишком много для образования прочных химических связей, так как ковалентный радиус атома алюминия равен $1.21 \AA$ [9]. Поэтому алюминий имеет низкий предел прочности и легко растрескивается, особенно под действием механических напряжений и в присутствии водорода, который дополнительно ослабляет связи. Слабые химические связи означают высокую скорость генерации вакансий $[6,10]$. Во-вторых, добавки сами должны образовывать прочные связи с атомами алюминия [10]. В чистом алюминии каждый атом имеет 12 ближайших соседей, поэтому желательно, чтобы атом добавки образовывал прочные связи с 12 атомами алюминия, не меняя всей структуры связей и не давая этим атомам возможности мигрировать в междоузлие даже при поддержке водорода. В-третьих, кристаллическая решетка со встроенными атомами добавки должна быть устойчивой. Например, если общая энергия системы будет уменьшаться при сближении двух атомов добавки в кристаллической решетке алюминия, то такая структура рано или поздно перейдет в более выгодную структуру, где заметная часть атомов алюминия будет слабо связана между собой и атомами добавки [10]. Переходы таких атомов в междоузлия будут порождать вакансии, что в конечном счете приведет к растрескиванию.

В настоящей работе для поиска добавок, удовлетворяющих всем трем требованиям, был выполнен прямой перебор элементов таблицы Менделеева от Li до Bi. C этой целью анализировались свойства модельной системы, представляющей собой суперьячейку из $3 \times 3 \times 3$ ячеек Al, состоящих из четырех атомов каждая. Таким образом, всего в исследуемой системе находилось 108 атомов [7]. Для моделирования свойств кристалла на суперьячейку накладывались периодические граничные условия по всем трем измерениям. Четыре атома 


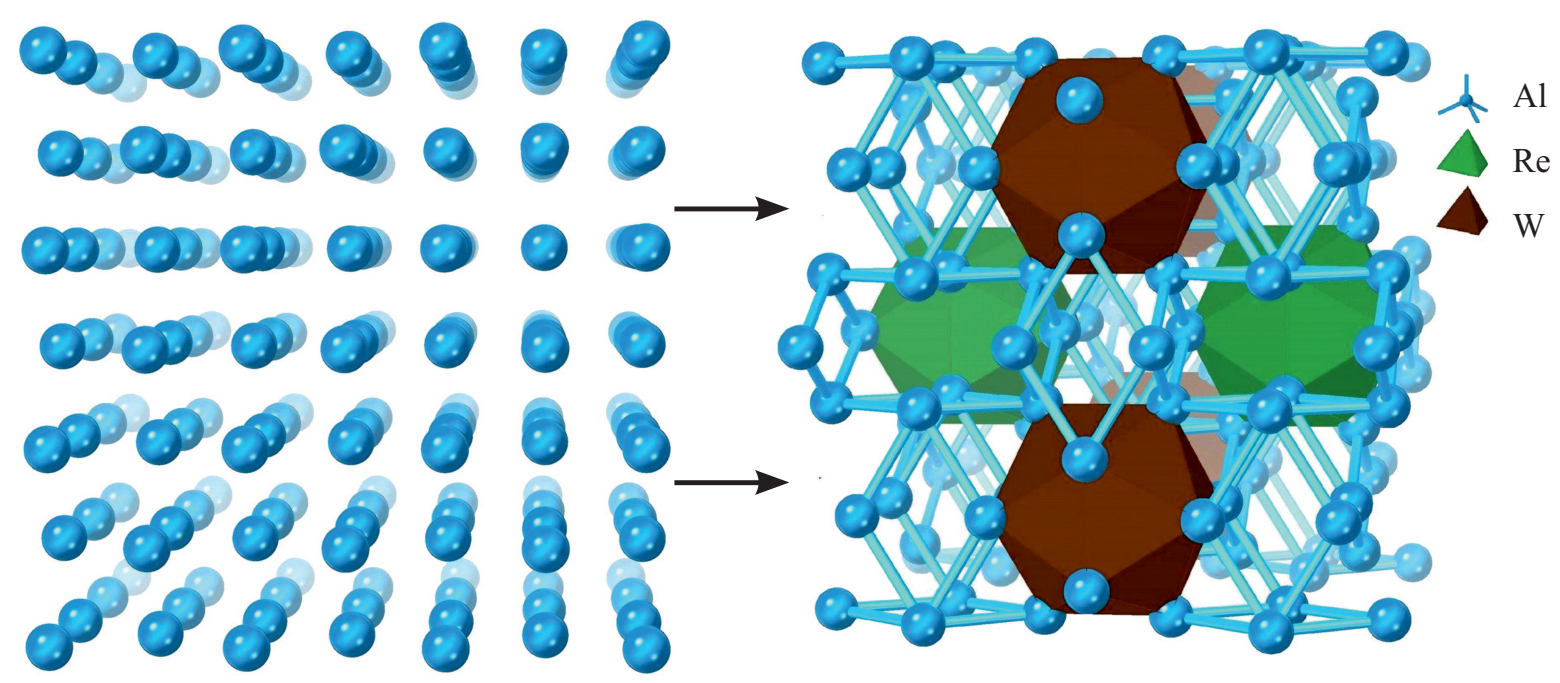

Pис. 1. Преобразование структуры атомов алюминия при замене двух атомов из 26 на атомы Re и W. Для наглядности атомы Re и $\mathrm{W}$ в соединении $\mathrm{WRe}_{24}$ представлены в виде полиэдров.

Al замещались атомами другого элемента так, чтобы сохранить симметрию системы $F m \overline{3} m$, т.е. в вершинах и центрах граней суперьячейки. Оптимизация геометрии системы выполнялась путем минимизации энергии по координатам всех атомов. Энергия вычислялась методом функционала плотности с использованием функционала PBE [11]. Параметры расчетов приведены в [6].

Результаты перебора элементов таковы. Лучше всего сжимает алюминий $\mathrm{Fe}$, т.е. в этом случае и размер суперъячейки, и длина связей между атомами $\mathrm{Al}$ минимальны (вблизи атомов $\mathrm{Fe}$ последняя равна $2.75 \AA$ ). Чуть хуже сжимают алюминий $\mathrm{Os}, \mathrm{Mn}$ и Со (длина связей $\mathrm{Al}$ $2.76 \AA$ ). Еще чуть хуже $\mathrm{Re}, \mathrm{Ru}, \mathrm{Cr}, \mathrm{Tc}, \mathrm{Ni}$ (длина связей Al $2.77 \AA$ ). В целом лучше всего сжимают Al элементы VIII группы (Fe, Os, Ru), затем VIIb группы ( $\mathrm{Mn}, \mathrm{Re}$, $\mathrm{Tc})$, затем VIb группы (Cr, W, Mo). Сравнивая между собой различные периоды таблицы Менделеева, можно заключить, что лучше сжимают алюминий элементы четвертого периода $(\mathrm{V}, \mathrm{Cr}, \mathrm{Mn}, \mathrm{Fe}, \mathrm{Co}, \mathrm{Ni})$, затем шестого периода (W, Re, Os, Ir).

Из всех перечисленных элементов самый большой ковалентный радиус у W $(1.62 \AA)$ и Мо $(1.54 \AA)$ [9], поэтому именно они образуют самые сильные связи с $\mathrm{Al}$, так как длина связей примерно одинакова и равна $2.80 \pm 0.05 \AA$. Далее идут $\mathrm{V}(1.52 \AA), \operatorname{Re}(1.51 \AA), \mathrm{Tc}$ $(1.47 \AA), \mathrm{Ru}(1.46 \AA), \mathrm{Os}(1.44 \AA)$ [10]. Из перечисленных элементов только W, Mo, Re и Tc образуют стабильные связи с 12 атомами $\mathrm{Al}$, удерживая их от миграции в междоузлие с образованием вакансии. Остальные элементы могут образовать стабильные связи лишь с меньшим числом атомов $\mathrm{Al}$, в результате часть атомов $\mathrm{Al}$ будет слабо связана, и на их месте могут образоваться вакансии, что в конечном счете может привести к зарождению трещин. У оставшихся элементов ковалентный радиус еще меньше, поэтому они связывают атомы $\mathrm{Al}$ еще менее эффективно. Кроме того, среди них также нет элементов, образующих стабильные связи с 12 атомами Al. Здесь необходимо отметить, что $\mathrm{W}$ относится к той же группе, что и Мо, но лучше сжимает алюминий и имеет больший ковалентный радиус, поэтому с точки зрения водородоустойчивости $\mathrm{W}$ лучше Мо по всем параметрам. Поскольку Тс не имеет стабильных изотопов, наиболее перспективными можно считать добавки Re и $\mathrm{W}$, а также смеси из них. Добавки этих элементов к $\mathrm{Al}$ приводят к образованию соединений $\mathrm{ReAl}_{12}, \mathrm{WAl}_{12}$. На практике важно избежать появления побочных фаз при растворении водорода в алюминиевом сплаве. В случае с $\mathrm{ReAl}_{12}, \mathrm{WAl}_{12}$ такими фазами являются $\mathrm{Al}, \mathrm{ReAl}_{6}, \mathrm{WAl}_{6}$, которые возникают при потере устойчивости исходной фазы из-за взаимодействия атомов $\mathrm{W} c \mathrm{~W}$ или $\mathrm{Re} \mathrm{c}$ Re. Общий рецепт ослабления взаимодействия хорошо известен: нужно, чтобы образовался твердый раствор $\mathrm{WReAl}_{24}$, тогда атомы $\mathrm{W}$ будут частично экранировать атомы Re, и наоборот. Указанный эффект исследован экспериментально для элементов этих же групп $\mathrm{Cr}$ и $\mathrm{Mn}$ [12]. Иными словами, твердый раствор $\mathrm{WReAl}_{24}$ должен быть более устойчив к изменению концентрации $\mathrm{W}$ и $\mathrm{Re}$, чем $\operatorname{ReAl}_{12}, \mathrm{WAl}_{12}$. Кроме того, $\mathrm{W}$ и $\mathrm{Re}$ превосходно дополняют друг друга с точки зрения водородоустойчивости. Re очень хорошо сжимает алюминий, связывая атомы алюминия между собой, а $\mathrm{W}$ отлично сам связывает атомы алюминия за счет большого ковалентного радиуса. Поэтому наиболее водородоустойчивым соединением алюминия можно считать $\mathrm{WReAl}_{24}$. Далее дан краткий анализ основных свойств $\mathrm{WReAl}_{24}$ с помощью методов квантовой химии с использованием функционала PBE.

Соединение $\mathrm{WReAl}_{24}$ может реализоваться в нескольких фазах, фаза с наименьшей энергией имеет кубическую симметрию $P m \overline{3}$ (рис. 1). Параметр решетки равен $7.56 \AA$, в кубической ячейке находятся 26 атомов, a именно 24 атома Al и по одному атому Re и W. 


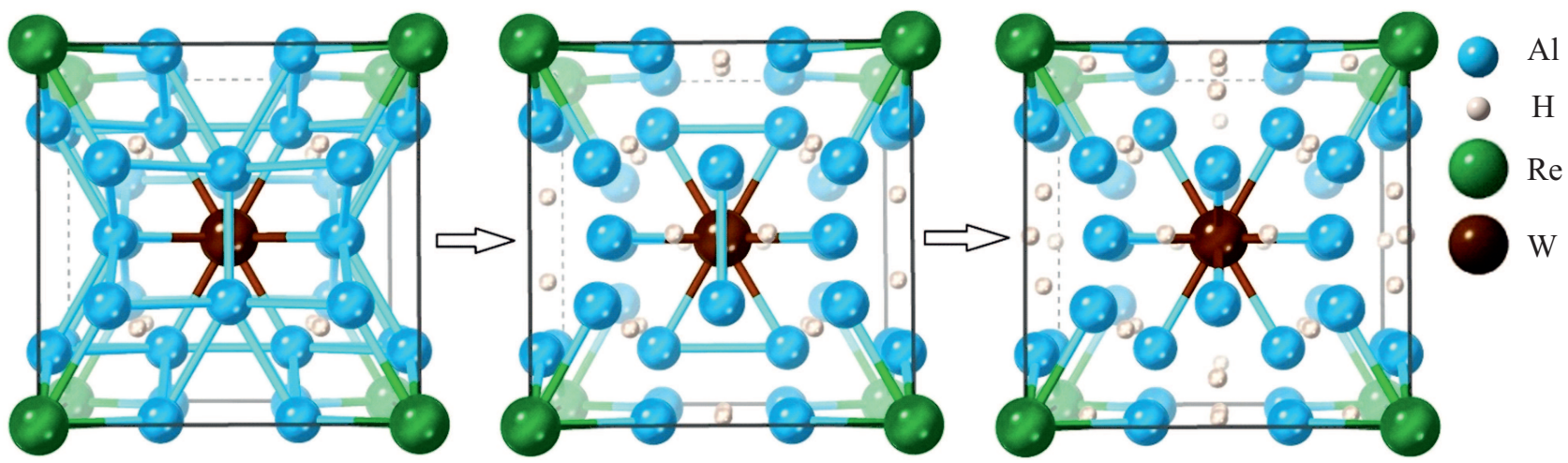

Рис. 2. Структура связей в соединении $\mathrm{WReAl}_{24}$ при растворении в нем водорода. Последовательно показана ячейка $\mathrm{WRe}_{24}$ из 26 атомов при наличии в ней $8,14,20$ атомов Н с сохранением симметрии $P m \overline{3}$. При растворении 8 атомов Н все связи между атомами $\mathrm{Al}$ еще сохраняются, при 14 атомах Н сохраняется лишь часть связей, при 20 атомах $\mathrm{H}$ атомы $\mathrm{Al}$ связаны только с атомами Re и W.
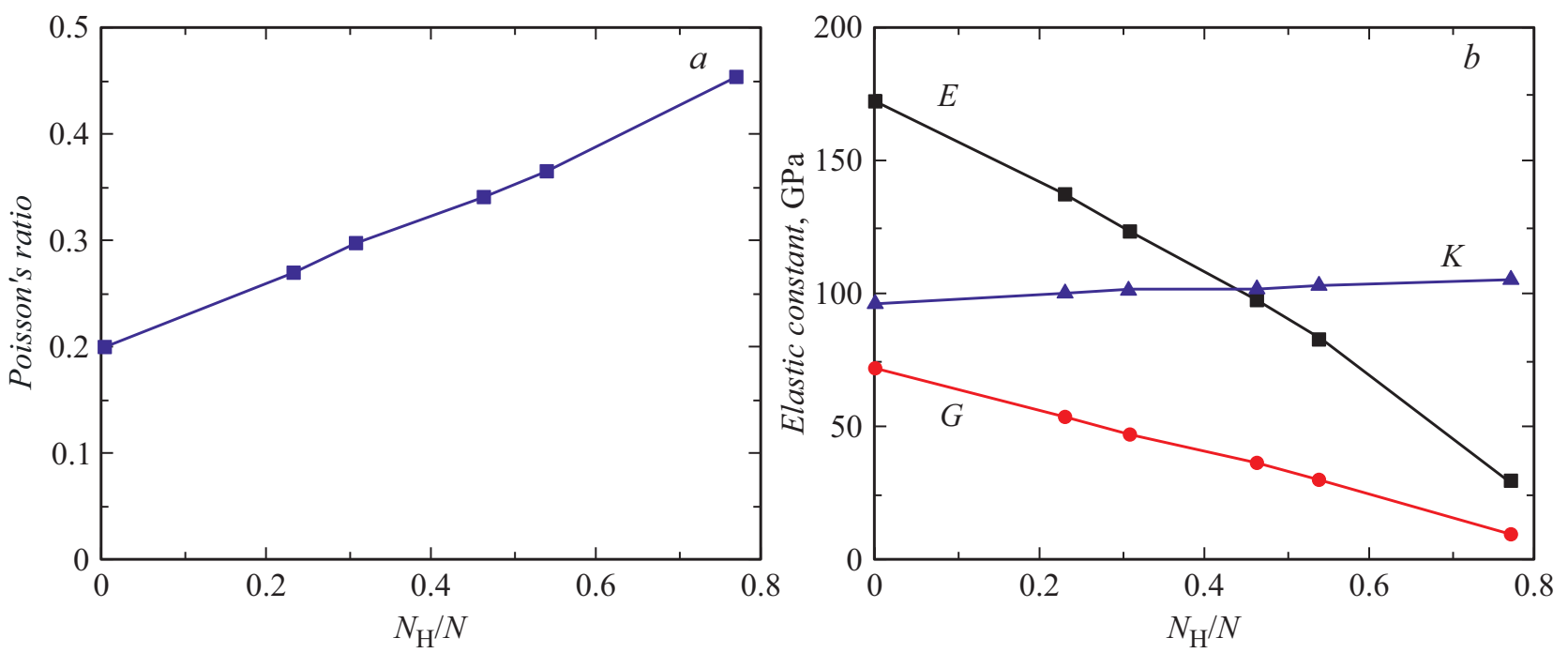

Рис. 3. Зависимости коэффициента Пуассона $(a)$, модуля Юнга $E$, коэффициента объемного сжатия $K$ и модуля сдвига $G$ соединения $\mathrm{WReAl}_{24}(b)$ от мольной концентрации водорода, растворенного в нем, т.е. от отношения $N_{\mathrm{H}} / N$, где $N=26-$ число атомов в исходной ячейке, $N_{\mathrm{H}}$ - число атомов водорода в одной ячейке $\mathrm{WReAl}_{24}$.

Плотность сплава равна $3.91 \mathrm{~g} / \mathrm{cm}^{3}$. Длина связей $\mathrm{Al}-\mathrm{Re}$ составляет $2.72 \AA, \mathrm{Al}-\mathrm{W}-2.73 \AA, \mathrm{Al}-\mathrm{Al}-2.77 \AA$, т. е. атомы алюминия достаточно сильно связаны как с добавками $\mathrm{Re}$ и $\mathrm{W}$, так и между собой за счет общего сжатия. Замена всего двух атомов из 26 приводит к кардинальному изменению структуры и свойств пустот в материале (рис. 1). В чистом Al на каждый атом приходится одна большая пустота радиусом $0.85 \AA$ и две маленькие радиусом $0.57 \AA$. Атомам Н выгодно находиться в маленьких пустотах, они связываются там с четырьмя атомами Al, длина связей $1.88 \AA$ [7]. Водород при этом сильно расширяет решетку $\mathrm{Al}$, ослабляя и без того слабые связи между атомами $\mathrm{Al}$ и делая материал более хрупким $[13,14]$. В одной ячейке соединения $\mathrm{WReAl}_{24}$ имеются 20 больших пустот радиусом $0.84 \AA$ и 40 маленьких радиусом $0.42 \AA$. Но водороду теперь выгодно находиться в больших пустотах, а не в маленьких, как в чистом Al. Расчет упругих характеристик соединения $\mathrm{WReAl}_{24}$ дает следующие результаты. Три независимые компоненты тензора упругости (с учетом кубической симметрии $P m \overline{3})$ равны $C_{11}=195 \mathrm{GPa}, C_{12}=47 \mathrm{GPa}$, $C_{44}=71 \mathrm{GPa}$, отсюда находятся средние значения модуля Юнга $E=173 \mathrm{GPa}$, модуля сдвига $G=72 \mathrm{GPa}$, модуля сжатия $K=96 \mathrm{GPa}$, коэффициента Пуассона $v=0.20$. У чистого алюминия расчет в этом же приближении дает $E_{\mathrm{Al}}=67 \mathrm{GPa}, G_{\mathrm{Al}}=25 \mathrm{GPa}, K_{\mathrm{Al}}=78 \mathrm{GPa}$, $v_{\mathrm{Al}}=0.36$, что очень хорошо совпадет с экспериментальными данными $E_{\text {exp }}=69 \mathrm{GPa}, G_{\exp }=26 \mathrm{GPa}$, $K_{\text {exp }}=77 \mathrm{GPa}, v_{\exp }=0.35[15]$, т.е. замена всего двух атомов из 26 делает материал заметно более жестким и твердым. Растворение водорода в сплаве расширяет решетку, ослабляя прежде всего связи между атомами алюминия [16] (рис. 2). Это существенно меняет упругие свойства сплава. На рис. 3 приведены зависимости 
упругих констант соединения $\mathrm{WReAl}_{24}$ от мольной концентрации растворенного в нем водорода, которые рассчитывались при добавлении в ячейку $N_{\mathrm{H}}=6,8,12,14$, 20 атомов водорода с сохранением исходной симметрии $P m \overline{3}$. Флуктуационная модель водородного охрупчивания сплава $\mathrm{WReAl}_{24}$ будет развита в отдельной публикации, в настоящей работе можно лишь отметить, что состояние $\mathrm{WReAl}_{24}$ с 16 атомами $\mathrm{H}$ в ячейке по своим упругопластическим свойствам примерно соответствует чистому Al.

Таким образом, в работе методами квантовой химии установлено, что добавки $\mathrm{W}$ и $\mathrm{Re}$ больше всего повышают водородоустойчивость $\mathrm{Al}$, т. е. замедляют процесс водородного охрупчивания за счет сжатия $\mathrm{Al}$ и усиления связей атомов Al между собой и с добавками. Рассчитаны основные физико-механические свойства $\mathrm{WReAl}_{24}$. Показано, что замена двух атомов Al из 26 на атомы $\mathrm{W}$ и $\mathrm{Re}$ примерно уравновешивает действие 16 атомов водорода.

\section{Финансирование работы}

Работа выполнена в рамках госзадания ФГУП ИПМаш PAH № AAAA-A18-118012790011-3.

\section{Конфликт интересов}

Авторы заявляют, что у них нет конфликта интересов.

\section{Список литературы}

[1] Aluminium alloys - recent trends in processing, characterization, mechanical behavior and applications, ed by S. Sivasankaran (InTech, Rijeka, 2017).

DOI: $10.5772 / 68032$

[2] L. Soler, J. Macanas, M. Munoz, J. Casado, J. Power Sources, 169, 144 (2007). DOI: 10.1016/j.jpowsour.2007.01.080

[3] J.B. von Colbe, J.-R. Ares, J. Barale, M. Baricco, C. Buckley, G. Capurso, N. Gallandat, D.M. Grant, M.N. Guzik, I. Jacob, E.H. Jensen, T. Jensen, J. Jepsen, T. Klassen, M.V. Lototskyy, K. Manickam, A. Montone, J. Puszkiel, S. Sartori, D.A. Sheppard, A. Stuart, G. Walker, C.J. Webb, H. Yang, V. Yartys, A. Züttel, M. Dornheim, Int. J. Hydrogen Energy, 44, 7780 (2019). DOI: 10.1016/j.jhydene.2019.01.104

[4] M. Nagumo, Fundamental of hydrogen embrittlement (Springer, Singapore, 2016). DOI: 10.1007/978-981-10-0161-1

[5] J.R. Scully, G.A. Young, S.W. Smith, in: Gaseous hydrogen embrittlement of materials in energy technologies, ed by R.P. Gangloff, B.P. Somerday (Woodhead Publishing, Ltd, Oxford, 2012), vol. 1, p. 707-768.

[6] Д.А. Индейцев, Е.В. Осипова, Письма в ЖТФ, 45 (17), 31 (2019). DOI: 10.21883/PJTF.2019.17.48221.17896

[7] Р.В. Регель, А.И. Слуцкер, Э.Е. Томашевский, Кинетическая природа прочности твердых тел (Наука, М., 1974).

[8] J.P. Perdew, K. Burke, M. Ernzerhof, Phys. Rev. Lett., 77, 3865 (1996). DOI: 10.1103/PhysRevLett.77.3865
[9] B. Cordero, V. Gomez, A.E. Platero-Prats, M. Reves, J. Echeverria, E. Cremades, F. Barragan, S. Alvarez, Dalton Trans., N 21, 2832 (2008). DOI: 10.1039/B801115J

[10] V.I. Kolesnikov, Yu F. Migal, I.V. Kolesnikov, E.S. Novikov, J. Friction Wear, 36, 1 (2015). DOI: $10.3103 / \mathrm{S} 1068366615010055$

[11] G. Kresse, J. Furthmuller, Phys. Rev. B, 54, 11169 (1996). DOI: $10.1103 /$ PhysRevB.54.11169

[12] L.F. Mondolfo, Aluminum alloys: structure and properties (Butterworth, London, 1976).

[13] Д.А. Индейцев, Е.В. Осипова, ФТТ, 51 (9), 1790 (2009).

[14] Д.А. Индейцев, Е.В. Осипова, ДАН, 440 (4), 472 (2011).

[15] G. Simmons, H. Wang, Single crystal elastic constants and calculated aggregate properties: a handbook (MIT Press, Cambridge, 1971).

[16] Д.А. Индейцев, Е.В. Осипова, ДАН, 484 (1), 56 (2019). DOI: $10.31857 / \mathrm{S} 0869-5652484156-60$ 